\title{
SOME ISOTROPIC CURVES AND REPRESENTATION IN COMPLEX SPACE $\mathbb{C}^{3}$
}

\author{
JinhuA QIAn AND Young Ho Kim
}

\begin{abstract}
In this paper, we give a representation formula for an isotropic curve with pseudo arc length parameter and define the structure function of such curves. Using the representation formula and the Frenet formula, the isotropic Bertrand curve and $k$-type isotropic helices are characterized in the 3 -dimensional complex space $\mathbb{C}^{3}$.
\end{abstract}

\section{Introduction}

In the 3 -dimensional complex space $\mathbb{C}^{3}$, the curves, of which the square of the distance between two points equal to zero, are called isotropic curves (see [4] and [7]). Due to the well-known one-to-one correspondence between the isotropic curves in $\mathbb{C}^{3}$ and the minimal surfaces in $\mathbb{R}^{3}$, the isotropic curves also can be understood as meromorphic mappings $r: t \in U \subset \mathbb{C} \rightarrow \mathbb{C}^{3}$, with vanishing complex length of their tangent vectors, i.e., with $\langle\dot{r}, \dot{r}\rangle=0$. Because of the close relation with minimal surfaces, isotropic curves are also called minimal curves.

The isotropic curves defined by a complex variable were deeply studied in recent years. For instance, U. Pekmen discussed the minimal space curves in the sense of Bertrand curves in [4], in which the author assume the two minimal curves have parallel tangents in opposite directions with common principal normals at corresponding points. S. Yilmaz and M. Turgut characterized the isotropic curves with constant pseudo curvature which is called the isotropic helix in [7]. In 2011, S. Yilmaz studied the $k$-type isotropic helices in $\mathbb{C}^{4}$ (see [6]), in which the sufficient and necessary conditions for each kind of isotropic helix are obtained by some differential equations of pseudo curvatures. However,

Received June 12, 2014; Revised September 12, 2014.

2010 Mathematics Subject Classification. 53A04, 53B30, 53C40.

Key words and phrases. isotropic curve, isotropic Bertrand curve, $k$-type isotropic helix, pesudo curvature, structure function.

The first author was supported by NSFC (No. 11371080).

The second author was supported by Basic Science Research Program through the National Research Foundation of Korea (NRF) funded by the Ministry of Education, Science and Technology (2012R1A1A2042298). 
due to the complication of some equations, some problems are restricted to the isotropic cubic, i.e., the isotropic curves have vanishing pseudo curvatures.

In this paper, we defined the isotropic Bertrand curves and $k$-type isotropic helices in $\mathbb{C}^{3}$. The sufficient and necessary conditions for an isotropic curve to be an isotropic Bertrand curve and a $k$-type isotropic helix are obtained. In addition, we define the structure function of isotropic curves. The relationship between the pseudo curvature and the structure function is also achieved. By solving some differential equations, the isotropic Bertrand curves and $k$-type isotropic helices in $\mathbb{C}^{3}$ are characterized.

In the present paper, $\mathbb{C}$ stands for the complex space and the isotropic curves under consideration are regular and analytic unless otherwise stated.

\section{Preliminaries}

Definition. The curves, of which the squared distance between two points equal to zero are called isotropic or minimal curves (see [4] and [7]).

Definition. A regular isotropic curve $r: t \in U \subset \mathbb{C} \rightarrow \mathbb{C}^{3}$ is full if and only if $\langle\ddot{r}, \ddot{r}\rangle \neq 0$.

To meet the requirements in the following discussion, the basic elements of isotropic curves in $\mathbb{C}^{3}$ are briefly presented.

Let $r(t): \mathbb{C} \rightarrow \mathbb{C}^{3}$ be an isotropic curve with the standard inner product

$$
\langle a, b\rangle=x_{1} y_{1}+x_{2} y_{2}+x_{3} y_{3}
$$

for $a=\left(x_{1}, x_{2}, x_{3}\right), b=\left(y_{1}, y_{2}, y_{3}\right) \in \mathbb{C}^{3}$. Recall that an arbitrary nonzero vector $v \in \mathbb{C}^{3}$ is called an isotropic vector if and only if $\langle v, v\rangle=0$.

According to the definition of isotropic curves, $\dot{r}(t)$ is an isotropic vector. Since it is impossible to normalize the tangent vector of an isotropic curve, we can adopt the so-called pseudo arc length parameter normalizing the acceleration vector such that $\left\langle r^{\prime \prime}(s), r^{\prime \prime}(s)\right\rangle=-1$ (for details, see [4] and [7]). Then, a curve is an isotropic curve if and only if

$$
d s^{2}=d r^{2}=0
$$

Remark. Hereafter, the isotropic curves are always assumed to be parametrized by the pseudo arc length.

Proposition 2.1. Let $r(s): \mathbb{C} \rightarrow \mathbb{C}^{3}$ be an isotropic curve. Then $r(s)$ can be framed by an unique Cartan Frenet frame $\left\{e_{1}, e_{2}, e_{3}\right\}$ such that

$$
\left\{\begin{array}{l}
r^{\prime}(s)=e_{1}(s) \\
e_{1}^{\prime}(s)=-i e_{2}(s) \\
e_{2}^{\prime}(s)=i \kappa(s) e_{1}(s)+i e_{3}(s) \\
e_{3}^{\prime}(s)=-i \kappa(s) e_{2}(s)
\end{array}\right.
$$

where

$$
\left\langle e_{1}, e_{1}\right\rangle=\left\langle e_{3}, e_{3}\right\rangle=\left\langle e_{1}, e_{2}\right\rangle=\left\langle e_{2}, e_{3}\right\rangle=0, \quad\left\langle e_{2}, e_{2}\right\rangle=\left\langle e_{1}, e_{3}\right\rangle=1 ;
$$


SOME ISOTROPIC CURVES AND REPRESENTATION IN COMPLEX SPACE $\mathbb{C}^{3} 965$

$e_{1} \wedge e_{2}=i e_{1}, e_{2} \wedge e_{3}=i e_{3}, e_{3} \wedge e_{1}=i e_{2} ; \quad \operatorname{det}\left(e_{1}, e_{2}, e_{3}\right)=i ; \quad i^{2}=-1$.

In sequel, $e_{1}, e_{2}, e_{3}$ are called the tangent, principal normal and binormal vector field of $r(s)$, respectively. The function

$$
\kappa(s)=\frac{1}{2}\left\langle r^{\prime \prime \prime}(s), r^{\prime \prime \prime}(s)\right\rangle
$$

is called the pseudo curvature of $r(s)$ (see [4]).

Next, we introduce the definitions of the isotropic Bertrand curves and the $k$-type isotropic helices, respectively.

Definition. Let $r(s)$ be an isotropic curve in $\mathbb{C}^{3}$. If there exists another isotropic curve $\tilde{r}(\tilde{s})$ in $\mathbb{C}^{3}$ such that the principal normal vector field of $\tilde{r}(\tilde{s})$ coincides with that of $r(s)$, then $r(s)$ is called an isotropic Bertrand curve and $\tilde{r}(\tilde{s})$ is called the isotropic Bertrand mate of $r(s)$ and vice versa.

Definition. Let $r(s)$ be an isotropic curve framed by $\left\{e_{1}, e_{2}, e_{3}\right\}$ in $\mathbb{C}^{3}$. If there exists a nonzero constant vector field $V \in \mathbb{C}^{3}$ such that $\left\langle e_{k}, V\right\rangle \neq 0(k=1,2,3)$ is a (complex) constant, then it is said to be a $k$-type $(k=1,2,3$, respectively) isotropic helix and $V$ is called the axis of $r(s)$.

\section{Representation formula of the isotropic curves in $\mathbb{C}^{3}$}

Let $r(s): \mathbb{C} \rightarrow \mathbb{C}^{3}$ be an isotropic curve. Since $r^{\prime}(s)$ is an isotropic vector, by putting $r^{\prime}(s)=\left(\xi_{1}(s), \xi_{2}(s), \xi_{3}(s)\right)$, then we have

$$
\xi_{1}^{2}+\xi_{2}^{2}+\xi_{3}^{2}=0 \text {. }
$$

From $\xi_{1}^{2}+\xi_{3}^{2}=-\xi_{2}^{2}$, we get

$$
\frac{\xi_{1}+i \xi_{3}}{-\xi_{2}}=\frac{\xi_{2}}{\xi_{1}-i \xi_{3}}
$$

Without loss of generality, we may assume

$$
\left\{\begin{array}{l}
\frac{\xi_{1}+i \xi_{3}}{\xi_{2}}=\frac{-\xi_{2}}{\xi_{1}-i \xi_{3}}=f(s), \\
\xi_{2}=2 \rho(s),
\end{array}\right.
$$

where $f(s)$ and $\rho(s)$ are analytic functions of complex variable $s$.

Rearranging (3.1), we get

$$
\left\{\begin{array}{l}
\xi_{1}+i \xi_{3}=2 \rho f, \\
\xi_{1}-i \xi_{3}=-2 \rho f^{-1} \\
\xi_{2}=2 \rho .
\end{array}\right.
$$

From (3.2), we have

$$
\left\{\begin{array}{l}
\xi_{1}=\rho\left(f-f^{-1}\right), \\
\xi_{2}=2 \rho \\
\xi_{3}=-i \rho\left(f+f^{-1}\right) .
\end{array}\right.
$$


Thus, the isotropic curve $r(s)$ can be written as

$$
r(s)=\int\left(\xi_{1}, \xi_{2}, \xi_{3}\right) d s=\int \rho\left(\left(f-f^{-1}\right), 2,-i\left(f+f^{-1}\right)\right) d s .
$$

From (3.3), after a simple calculation, we have

$$
r^{\prime \prime}(s)=\rho_{s}\left(\left(f-f^{-1}\right), 2,-i\left(f+f^{-1}\right)\right)+\rho f_{s}\left(1+f^{-2}, 0,-i\left(1-f^{-2}\right)\right) .
$$

Since $s$ is the pseudo arc length of $r(s)$, from the above equation, we have

$$
\left\langle r^{\prime \prime}(s), r^{\prime \prime}(s)\right\rangle=4 \rho^{2} f_{s}^{2} f^{-2}=-1 .
$$

Obviously, $f_{s}=\frac{d f}{d s} \neq 0$. By an appropriate transformation, if necessary, we can assume

$$
\rho(s)=\frac{i f(s)}{2 f_{s}(s)} .
$$

Substituting (3.4) into (3.3), we obtain:

Theorem 3.1. Let $r(s): \mathbb{C} \rightarrow \mathbb{C}^{3}$ be an isotropic curve. Then $r(s)=$ $\left(x_{1}(s), x_{2}(s), x_{3}(s)\right)$ can be written as

$$
r(s)=\frac{i}{2} \int f_{s}^{-1}\left(\left(f^{2}-1\right), 2 f,-i\left(f^{2}+1\right)\right) d s
$$

or

$$
\left\{\begin{array}{l}
x_{1}(s)=i \int\left(\frac{f^{2}(s)-1}{2 f_{s}(s)}\right) d s, \\
x_{2}(s)=i \int\left(\frac{f(s)}{f_{s}(s)}\right) d s, \\
x_{3}(s)=\int\left(\frac{f^{2}(s)+1}{2 f_{s}(s)}\right) d s
\end{array}\right.
$$

for some non constant analytic function $f(s)$.

Definition. The function $f(s)$ in Theorem 3.1 is called the structure function of the isotropic curve $r(s)$ with pseudo arc length parameter $s$.

In the following, we discuss the relation between the pseudo curvature and the structure function. From Theorem 3.1, through direct calculation, we have

$$
\begin{aligned}
r^{\prime \prime \prime}(s)= & \left(f_{s}^{-3} f_{s s}^{2}-\frac{1}{2} f_{s}^{-2} f_{s s s}\right)\left(i\left(f^{2}-1\right), 2 i f,\left(f^{2}+1\right)\right) \\
& -f_{s}^{-1} f_{s s}(i f, i, f)+f_{s}(i, 0,1) .
\end{aligned}
$$

Based on $\kappa(s)=\frac{1}{2}\left\langle r^{\prime \prime \prime}(s), r^{\prime \prime \prime}(s)\right\rangle$ and the above equation, we have:

Theorem 3.2. The pseudo curvature $\kappa(s)$ and the structure function $f(s)$ of an isotropic curve satisfy

$$
\kappa(s)=-S(f)(s)
$$

where

$$
(S f)(s)=\left(\frac{f_{s s}}{f_{s}}\right)_{s}-\frac{1}{2}\left(\frac{f_{s s}}{f_{s}}\right)^{2}
$$


is the Schwarzian derivative of the function $f(s)$ (see [5]).

Remark. Using Theorem 3.1 and Theorem 3.2, we can study the properties of the isotropic curves and construct isotropic curves with certain characters.

First, we consider the isotropic curves with vanishing pseudo curvatures. In such a case, by the Frenent formula (2.1), we have

$$
r^{(4)}(s)=0 .
$$

Solving the above equation and by an appropriate translation, we have:

Theorem 3.3. Let $r(s)$ be an isotropic curve with vanishing pseudo curvature. Then it is an isotropic cubic written by

$$
r(s)=C_{1} s^{3}+C_{2} s^{2}+C_{3} s,
$$

where $C_{1}, C_{2}, C_{3} \in \mathbb{C}^{3}$.

Corollary 3.4. Let $r(s)$ be an isotropic curve with vanishing pseudo curvature. Then the structure function $f(s)$ of $r(s)$ is given by

$$
f(s)=\frac{c_{1}}{s+c_{2}}
$$

where $c_{1}, c_{2} \in \mathbb{C}$ and $c_{1} \neq 0$.

Proof. From Theorem 3.2, when $\kappa \equiv 0$, we have

$$
\left(\frac{f_{s s}}{f_{s}}\right)_{s}-\frac{1}{2}\left(\frac{f_{s s}}{f_{s}}\right)^{2}=0 .
$$

We put

$$
\frac{f_{s s}}{f_{s}}=g(s)
$$

Substituting (3.7) into (3.6), we have

$$
2 g^{\prime}(s)=g^{2}(s)
$$

Then, we get

$$
g(s)=\frac{-2}{s+c},
$$

where $c \in \mathbb{C}$ is a constant of integration.

Substituting (3.9) into (3.7) and integrating twice, we get the conclusion.

Next, we consider the case that the isotropic curve has a nonzero constant pseudo curvature. From the Frenent formula (2.1), we have

$$
r^{(4)}(s)=2 \kappa(s) r^{\prime \prime}(s) .
$$

Putting $r^{\prime \prime}(s)=\omega(s)$, then the above equation can be written as

$$
\omega^{\prime \prime}(s)=2 \kappa(s) \omega(s) .
$$


Since $\kappa(s)$ is a nonzero constant, without loss of generality, we may assume

$$
2 \kappa=r(\cos \theta+i \sin \theta),
$$

where $r, \theta \in \mathbb{R}$ and $r>0, \theta \in[0,2 \pi)$. Meanwhile, we may put

$$
\omega(s)=\omega(x+i y)=u(x, y)+i v(x, y),
$$

where $u, v$ are analytic functions. Then, we have

$$
\omega^{\prime \prime}(s)=u_{x x}+i v_{x x}=-\left(u_{y y}+i v_{y y}\right) .
$$

Substituting (3.11)-(3.13) into (3.10), we have

$$
\left\{\begin{array}{l}
-u_{y y}=u_{x x}=r \cos \theta u-r \sin \theta v, \\
-v_{y y}=v_{x x}=r \sin \theta u+r \cos \theta v .
\end{array}\right.
$$

Putting

$$
Y=\left(\begin{array}{l}
u \\
v
\end{array}\right), \quad A=\left(\begin{array}{cc}
r \cos \theta & -r \sin \theta \\
r \sin \theta & r \cos \theta
\end{array}\right) .
$$

Then, the system of partial differential equations (3.14) can be written as

$$
\left\{\begin{array}{l}
\frac{\partial^{2}}{\partial x^{2}} Y=A Y \\
\frac{\partial^{2}}{\partial y^{2}} Y=-A Y .
\end{array}\right.
$$

Solving (3.15), we get

$$
Y=C \exp (B x) \exp (i B y)=C \exp (B s),
$$

where

$$
C=\left(\begin{array}{l}
c_{1} \\
c_{2}
\end{array}\right), \quad B=\left(\begin{array}{cc}
\sqrt{r} \cos \frac{\theta}{2} & -\sqrt{r} \sin \frac{\theta}{2} \\
\sqrt{r} \sin \frac{\theta}{2} & \sqrt{r} \cos \frac{\theta}{2}
\end{array}\right),
$$

$c_{1}, c_{2}$ are constants and $B^{2}=A$.

For convenience, we denote by

$$
\left\{\begin{array}{l}
\sqrt{r} \cos \frac{\theta}{2}=\alpha \\
\sqrt{r} \sin \frac{\theta}{2}=\beta .
\end{array}\right.
$$

According to the property of exponential function of matrix (see [2]), we have

$$
\exp (B s)=e^{\alpha s}\left(\begin{array}{cc}
\cos \beta s & -\sin \beta s \\
\sin \beta s & \cos \beta s
\end{array}\right) .
$$

Thus, we have

$$
Y=e^{\alpha s}\left(\begin{array}{l}
c_{1} \cos \beta s-c_{2} \sin \beta s \\
c_{1} \sin \beta s+c_{2} \cos \beta s
\end{array}\right)
$$

Consequently, $\omega(s)$ is obtained by

$$
\omega(s)=e^{\alpha s}\left[\left(c_{1} \cos \beta s-c_{2} \sin \beta s\right)+i\left(c_{1} \sin \beta s+c_{2} \cos \beta s\right)\right] .
$$

Integrating (3.16) with respect to $s$ twice, we obtain: 
Theorem 3.5. Let $r(s)$ be an isotropic curve with a nonzero constant pseudo curvature

$$
\kappa=\frac{r}{2}(\cos \theta+i \sin \theta) \quad(r>0, \theta \in[0,2 \pi)) .
$$

Then $r(s)$ can be expressed by

$$
r(s)=\frac{C_{1}}{r}(\cos \theta-i \sin \theta) e^{\alpha s}(\cos \beta s+i \sin \beta s)+C_{2} s+C_{3},
$$

where $\alpha=\sqrt{r} \cos \frac{\theta}{2}, \beta=\sqrt{r} \sin \frac{\theta}{2}$ and $C_{1}, C_{2}, C_{3} \in \mathbb{C}^{3}$.

Remark. The representation forms of isotropic curves presented by Theorem 3.3 and Theorem 3.5 will be often used in the discussion from now on.

\section{Isotropic Bertrand curves in $\mathbb{C}^{3}$}

In this section, we mainly discuss the sufficient and necessary condition for an isotropic curve to be an isotropic Bertrand curve.

Theorem 4.1. Let $r(s)$ be an isotropic curve in $\mathbb{C}^{3}$. Then it is an isotropic Bertrand curve if and only if it has a nonzero constant pseudo curvature.

Proof. Suppose that $(r(s), \tilde{r}(\tilde{s}))$ is an isotropic Bertrand pair. Then $\tilde{r}(\tilde{s})$ can be expressed by

$$
\tilde{r}(\tilde{s})=r(s)+\lambda(s) e_{2}(s),
$$

where $\lambda(s)$ is a nonzero analytic function and $\tilde{s}$ is the pseudo arc length parameter of $\tilde{r}(\tilde{s})$.

Assume that $r(s)$ and $\tilde{r}(\tilde{s})$ are framed by $\left\{e_{1}, e_{2}, e_{3}\right\}$ and $\left\{\tilde{e}_{1}, \tilde{e}_{2}, \tilde{e}_{3}\right\}$, respectively. Differentiating (4.1) with respect to $s$, we have

$$
\tilde{e}_{1} \frac{d \tilde{s}}{d s}=(1+i \lambda \kappa) e_{1}+\lambda^{\prime} e_{2}+i \lambda e_{3},
$$

Since $\tilde{e}_{2}=\varepsilon e_{2}(\varepsilon= \pm 1)$, by taking the scalar product with (4.2), we have $\lambda^{\prime}=0$. Thus, $\lambda$ is a nonzero constant and (4.2) can be written as

$$
\tilde{e}_{1} \frac{d \tilde{s}}{d s}=(1+i \lambda \kappa) e_{1}+i \lambda e_{3} .
$$

Taking the scalar product to both sides of (4.3), we obtain

$$
2 i \lambda(1+i \lambda \kappa)=0 \text {. }
$$

So, the pseudo curvature $\kappa(s)=\frac{i}{\lambda}$ is a nonzero constant.

Conversely, for an isotropic curve $r(s)$ with a nonzero constant pseudo curvature, we can define a new curve $\tilde{r}(\tilde{s})$ as following:

$$
\tilde{r}(\tilde{s})=r(s)+\frac{i}{\kappa} e_{2}(s) .
$$

Obviously, it is an isotropic curve.

Differentiating (4.4) with respect to $s$, we get

$$
\tilde{e}_{1} \frac{d \tilde{s}}{d s}=-\frac{e_{3}}{\kappa} .
$$


Differentiating (4.5) with respect to $s$, we obtain

$$
-i \tilde{e}_{2}\left(\frac{d \tilde{s}}{d s}\right)^{2}+\tilde{e}_{1} \frac{d^{2} \tilde{s}}{d s^{2}}=i e_{2} .
$$

Taking the scalar product to both sides of (4.6) with $e_{2}$ and $\tilde{e}_{2}$, respectively, we have

$$
\left\{\begin{array}{l}
-i\left\langle e_{2}, \tilde{e}_{2}\right\rangle\left(\frac{d \tilde{s}}{d s}\right)^{2}+\left\langle e_{2}, \tilde{e}_{1}\right\rangle \frac{d^{2} \tilde{s}}{d s^{2}}=i, \\
-i\left\langle\tilde{e}_{2}, \tilde{e}_{2}\right\rangle\left(\frac{d \tilde{s}}{d s}\right)^{2}=i\left\langle e_{2}, \tilde{e}_{2}\right\rangle .
\end{array}\right.
$$

From (4.5), we have $\left\langle e_{2}, \tilde{e}_{1}\right\rangle=0$. Then, (4.7) yields

$$
\left\langle e_{2}, \tilde{e}_{2}\right\rangle=\frac{1}{\left\langle e_{2}, \tilde{e}_{2}\right\rangle} \text {. }
$$

Because $e_{2}$ and $\tilde{e}_{2}$ are all real vectors, (4.8) implies that $e_{2}=\varepsilon \tilde{e}_{2}(\varepsilon= \pm 1)$. The curve $\tilde{r}(\tilde{s})$ is the isotropic Bertrand mate of $r(s)$. This completes the proof.

From Theorem 4.1 and Theorem 3.5, we can characterize the isotropic Bertrand curves as follows:

Corollary 4.2. Let $r(s)$ be an isotropic Bertrand curve in $\mathbb{C}^{3}$. Then $r(s)$ can be expressed by (3.17).

\section{5. $k$-type isotropic helices in $\mathbb{C}^{3}$}

In this section, we discuss three kinds of isotropic helices. In order to facilitate the discussion below, we make some notations and calculations, firstly.

Let $V$ be an axis of a $k$-type isotropic helix. Taking into account of the Cartan Frenet frame used in Proposition 2.1, it can be decomposed by

$$
V=v_{1} e_{1}(s)+v_{2} e_{2}(s)+v_{3} e_{3}(s),
$$

where $v_{i}=v_{i}(s)(i=1,2,3)$ are analytic functions of pseudo arc length $s$ and

$$
v_{1}=\left\langle e_{3}, V\right\rangle, \quad v_{2}=\left\langle e_{2}, V\right\rangle, \quad v_{3}=\left\langle e_{1}, V\right\rangle .
$$

Differentiating (5.1) with respect to $s$, we get

$$
\left(v_{1}^{\prime}+i \kappa v_{2}\right) e_{1}+\left(v_{2}^{\prime}-i v_{1}-i \kappa v_{3}\right) e_{2}+\left(v_{3}^{\prime}+i v_{2}\right) e_{3}=0,
$$

which implies

$$
\left\{\begin{array}{l}
v_{1}^{\prime}+i \kappa v_{2}=0, \\
v_{2}^{\prime}-i v_{1}-i \kappa v_{3}=0, \\
v_{3}^{\prime}+i v_{2}=0
\end{array}\right.
$$

We now consider:

Theorem 5.1. Let $r(s)$ be an isotropic curve in $\mathbb{C}^{3}$. Then the following statements are equivalent:

(1) $r(s)$ is a 1-type isotropic helix.

(2) $r(s)$ is a 3-type isotropic helix.

(3) The pseudo curvature of $r(s)$ is a constant. 
Proof. At first, according to the definition of 1-type isotropic helix, we have

$$
v_{3}=\left\langle e_{1}, V\right\rangle=C_{0},
$$

where $C_{0}$ is a nonzero constant. Substituting it into (5.2), we get

$$
\left\{\begin{array}{l}
v_{1}^{\prime}+i \kappa v_{2}=0, \\
v_{2}^{\prime}-i v_{1}-i \kappa C_{0}=0, \\
i v_{2}=0
\end{array}\right.
$$

Equation (5.3) yields

Obviously, $\kappa=$ constant.

$$
\left\{\begin{array}{l}
v_{1}^{\prime}=v_{2}=0 \\
v_{1}+\kappa C_{0}=0
\end{array}\right.
$$

Similarly, based on the definition of 3-type isotropic helix, we have

$$
v_{1}=\left\langle e_{3}, V\right\rangle=C_{0},
$$

where $C_{0}$ is a nonzero constant. Substituting it into (5.2), we get

$$
\left\{\begin{array}{l}
i \kappa v_{2}=0 \\
v_{2}^{\prime}-i C_{0}-i v_{3} \kappa=0 \\
i v_{2}+v_{3}^{\prime}=0
\end{array}\right.
$$

By the first equation in (5.4), we consider the open subset $\mathcal{O}=\{p \in r \mid \kappa(p) \neq$ $0\}$ and assume $\mathcal{O} \neq \emptyset$. Then, we have

$$
\left\{\begin{array}{l}
v_{3}^{\prime}=v_{2}=0 \\
\kappa v_{3}+C_{0}=0
\end{array}\right.
$$

By (5.5), obviously, $\kappa$ is a constant.

On the other hand, when $\mathcal{O}=\emptyset$, then the pseudo curvature $\kappa \equiv 0$. By continuity, the pseudo curvature $\kappa(s)$ is a constant for 3-type isotropic helices.

Conversely, suppose that $r(s)$ is an isotropic curve with constant pseudo curvature. By choosing the vector field $V$ as following:

$$
V=c\left(-\kappa e_{1}+e_{3}\right), \quad(c \in \mathbb{C}-\{0\}),
$$

we have $V^{\prime}=0$ and $\left\langle e_{1}, V\right\rangle=c$. So $V$ is a constant vector and $r(s)$ is a 1-type isotropic helix.

At the same time, we can choose the vector fields $V$ as

$$
V=c\left(e_{1}-\frac{1}{\kappa} e_{3}\right), \quad(c \in \mathbb{C}-\{0\})
$$

for $\kappa \neq 0$ and

(5.8) $V=c\left[e_{1}+\left(i s+c_{1}\right) e_{2}+\left(\frac{1}{2} s^{2}-i c_{1} s+c_{2}\right) e_{3}\right], \quad\left(c \in \mathbb{C}-\{0\}, c_{1}, c_{2} \in \mathbb{C}\right)$ for $\kappa=0$.

In both cases, we have $V^{\prime}=0$ and $\left\langle e_{3}, V\right\rangle=c$. Thus, $V$ is a constant vector and $r(s)$ is a 3-type isotropic helix. This completes the proof.

As an immediate consequence of Theorem 5.1, we have: 
Corollary 5.2. Let $r(s)$ be a 1-type isotropic helix in $\mathbb{C}^{3}$. Then the axes of $r(s)$ are taken as (5.6).

Corollary 5.3. Let $r(s)$ be a 3-type isotropic helix in $\mathbb{C}^{3}$. Then the axes of $r(s)$ can be expressed by (5.8) when $\kappa=0$ and represented by (5.7) when $\kappa$ is a nonzero constant.

From Theorem 5.1, Theorem 3.3 and Theorem 3.5, we can characterize the 1-type and 3-type isotropic helices in the following:

Corollary 5.4. Let $r(s)$ be a 1-type (3-type) isotropic helix in $\mathbb{C}^{3}$. Then, when the pseudo curvature vanishes, $r(s)$ can be expressed by $(3.5)$; when the pseudo curvature is a nonzero constant, $r(s)$ is denoted by (3.17).

Summarizing Theorem 4.1 and Theorem 5.1, we easily have:

Theorem 5.5. Let $r(s)$ be an isotropic curve with a nonzero constant pseudo curvature in $\mathbb{C}^{3}$. Then the following statements are equivalent:

(1) $r(s)$ is an isotropic Bertrand curve.

(2) $r(s)$ is a 1-type isotropic helix.

(3) $r(s)$ is a 3-type isotropic helix.

At last, we discuss the 2-type isotropic helix.

Theorem 5.6. Let $r(s)$ be an isotropic curve in $\mathbb{C}^{3}$. Then $r(s)$ is a 2-type isotropic helix if and only if the pseudo curvature is expressed by

$$
\kappa(s)=\frac{a}{(s+b)^{2}},
$$

where $a, b \in \mathbb{C}$.

Proof. From the definition of 2-type isotropic helix, we have

$$
v_{2}=\left\langle e_{2}, V\right\rangle=C_{0},
$$

where $C_{0}$ is a nonzero constant. Substituting it into (5.2), we get

$$
\left\{\begin{array}{l}
v_{1}^{\prime}+i \kappa C_{0}=0, \\
v_{1}+\kappa v_{3}=0, \\
i C_{0}+v_{3}^{\prime}=0,
\end{array}\right.
$$

from which, we get

i.e.,

$$
\left(\frac{\kappa}{\kappa^{\prime}}\right)^{\prime}=-\frac{1}{2},
$$

$$
2 \kappa \kappa^{\prime \prime}-3 \kappa^{2}=0 .
$$

Solving it, we obtain

$$
\kappa(s)=\frac{a}{(s+b)^{2}},
$$

where $a, b \in \mathbb{C}$. 
Conversely, suppose $r(s)$ is an isotropic curve satisfying (5.10). By choosing the vector field $V$ as follows:

$$
V=c\left[\left(-i \int \kappa d s\right) e_{1}+e_{2}+\left(i \frac{\int \kappa d s}{\kappa}\right) e_{3}\right], \quad(c \in \mathbb{C}-\{0\}),
$$

we have $V^{\prime}=0$ and $\left\langle e_{2}, V\right\rangle=c$. Thus, $V$ is a constant vector and $r(s)$ is a 2-type isotropic helix.

Corollary 5.7. Let $r(s)$ be a 2-type isotropic helix in $\mathbb{C}^{3}$. Then the axes of $r(s)$ are obtained by (5.12).

In the following, we try to express the 2-type isotropic helices. By an appropriate parameter transformation, we may put $b=0$ in $(5.11)$. Then, the pseudo curvature $\kappa(s)=a s^{-2}$. By the Frenet formulas (2.1), we get

$$
s^{3} e_{1}^{\prime \prime \prime}(s)-2 a s e_{1}^{\prime}(s)+2 a e_{1}(s)=0,
$$

where $a \in \mathbb{C}$. Denoting $r^{\prime}(s)=e_{1}(s)$, we have:

Theorem 5.8. Let $r(s)$ be a 2-type isotropic helix in $\mathbb{C}^{3}$. Then the position vector of $r(s)$ satisfies

$$
s^{3} r^{(4)}(s)-2 a s r^{\prime \prime}(s)+2 a r^{\prime}(s)=0,
$$

where $a \in \mathbb{C}$.

The vector differential equation in Theorem 5.8 is a characterization for the 2-type isotropic helix. However, the general solution has not been found yet. In particular, when the tangent vector $e_{1}(s)$ is parametrized by a real variable $s$ and $a$ is a real number, by solving the Euler equation (5.13), we obtain the following three cases:

(1) $e_{1}(s)=B_{1} s+B_{2} s \log s+B_{3} s \log ^{2} s$ for $2 a=-1$;

(2) $e_{1}(s)=B_{1} s+B_{2} s^{(1+\sqrt{1+2 c})}+B_{3} s^{(1-\sqrt{1+2 c})}$ for $2 a>-1$;

(3) $e_{1}(s)=B_{1} s+B_{2} s \sin [(\sqrt{-1-2 c}) \log s]+B_{3} s \cos [(\sqrt{-1-2 c}) \log s]$ for $2 a<-1$,

where $B_{i}(i=1,2,3) \in \mathbb{C}^{3}$.

Therefore, we have:

Corollary 5.9. Let $r(s): \mathbb{R} \rightarrow \mathbb{C}^{3}$ be a 2-type isotropic helix in $\mathbb{C}^{3}$ and the pseudo curvature $\kappa(s)=a s^{-2}(a \in \mathbb{R})$. Then $r(s)$ can be written by

(1) $r(s)=C_{1} s^{2}+C_{2} s^{2} \log s+C_{3} s^{2} \log ^{2} s$ for $2 a=-1$;

(2) $r(s)=C_{1} s^{2}+C_{2} s^{(2+\sqrt{1+2 a})}+C_{3} s^{(2-\sqrt{1+2 a})}$ for $2 a>-1$;

(3) $r(s)=C_{1} s^{2}+C_{2} s^{2} \sin [(\sqrt{-1-2 a}) \log s]+C_{3} s^{2} \cos [(\sqrt{-1-2 a}) \log s]$ for $2 a<-1$,

where $C_{i}(i=1,2,3) \in \mathbb{C}^{3}$.

Corollary 5.10. Let $r(s): \mathbb{R} \rightarrow \mathbb{C}^{3}$ be a 2-type isotropic helix in $\mathbb{C}^{3}$ and the pseudo curvature $\kappa(s)=a s^{-2}(a \in \mathbb{R})$. Then the structure function $f(s)$ of $r(s)$ is given by 
(1) $f(s)=s^{c}$ or $f(s)=s^{-c}$ for $c \neq 0, \pm 1$ and $2 a=c^{2}-1$;

(2) $f(s)=\frac{c}{\log s}$ or $f(s)=\frac{\log s}{c}$ for $c \neq 0$ and $2 a=-1$;

(3) $f(s)=\frac{2}{c} \tan \left(\frac{c}{2} \log s\right)$ or $f(s)=-\frac{2}{c} \tan ^{-1}\left(\frac{c}{2} \log s\right)$ for $c \neq 0$ and $2 a=$ $-c^{2}-1$,

where $a, c \in \mathbb{R}$.

Proof. From Theorem 3.2, we have

$$
\left(\frac{f_{s s}}{f_{s}}\right)_{s}-\frac{1}{2}\left(\frac{f_{s s}}{f_{s}}\right)^{2}=-a s^{-2} .
$$

Similarly to the proof of Corollary 3.4 , we can put

$$
\frac{f_{s s}}{f_{s}}=g(s) \text {. }
$$

Substituting (5.15) into (5.14), we have

$$
g^{\prime}(s)=\frac{1}{2} g^{2}(s)-\frac{a}{s^{2}} .
$$

Solving the above Ricatti equation (5.16) and by an appropriate transformation, we can get the conclusion (see [3]).

Acknowledgment. The authors would like to express their thanks to the referee suggesting some valuable points which help the paper to be improved.

\section{References}

[1] A. Ali, R. López, and M. Turgut, $k$-type partially null and pseudo null slant helices in Minkowski 4-space, Math. Commun. 17 (2012), no. 1, 93-103.

[2] R. A. Horn and C. R. Johnson, Topics in Matrix Analysis, Cambridge University Press, 1991.

[3] H. Liu and Q. Meng, Representation formulas of curves in a two- and three-dimensional lightlike cone, Results Math. 59 (2011), no. 3-4, 437-451.

[4] U. Pekmen, On minimal space curves in the sense of Bertrand curves, Univ. Beograd. Publ. Elektrotehn. Fak. Ser. Mat. 10 (1999), 3-8.

[5] B. Schwarz, Complex nonoscillation theorems and criteria of univalence, Trans. Amer. Math. Soc. 80 (1955), 159-186.

[6] S. Yilmaz, Contributions to differential geometry of isotropic curves in the complex space, J. Math. Anal. Appl. 374 (2011), no. 2, 673-680.

[7] S. Yilmaz and M. Turgut, Some characterizations of isotropic curves in the Euclidean space, Int. J. Comput. Math. Sci. 2 (2008), no. 2, 107-109.

JiNHUA QIAN

Department of Mathematics

NORTHEASTERN UNIVERSITY

Shenyang 110004, P. R. China

E-mail address: ruoyunqian@163.com 
SOME ISOTROPIC CURVES AND REPRESENTATION IN COMPLEX SPACE $\mathbb{C}^{3} 975$

Young Ho KIM

Department of Mathematics

Kyungpook National University

DAEGU 702-701, Korea

E-mail address: yhkim@knu.ac.kr 